\title{
Composition of cuticular lipids in the pteromalid wasp Lariophagus distinguendus is host dependent
}

\author{
S. Kühbandner ${ }^{1}$, K. Hacker ${ }^{2}$, S. Niedermayer ${ }^{2}$, \\ J.L.M. Steidle ${ }^{2}$ and J. Ruther ${ }^{1 *}$ \\ ${ }^{1}$ Institute of Zoology, University of Regensburg, Universitätsstraße 31, 93053 \\ Regensburg, Germany: ${ }^{2}$ Institute for Zoology, University of Hohenheim, \\ Garbenstraße 30, 70599 Stuttgart, Germany
}

\begin{abstract}
The insect cuticle is covered by a thin layer of hydrocarbons not only preventing desiccation but also playing an important role in the sexual communication of several species. In the pteromalid wasp Lariophagus distinguendus, a parasitoid of grain infesting beetles, female cuticular hydrocarbons (CHCs) elicit male courtship behaviour. We analyzed the CHC profiles of male and female L. distinguendus wasps reared on different beetle hosts by coupled gas chromatography- mass spectrometry (GC-MS). Statistical analysis of the data revealed significant differences between strains reared on different hosts, while spatially isolated strains reared on the same host produced similar profiles. CHC profiles of parasitoids reared on Stegobium paniceum were statistically distinguishable from those of wasps reared on all other hosts. A host shift from Sitophilus granarius to S. paniceum resulted in distinguishable $\mathrm{CHC}$ profiles of L. distinguendus females after only one generation. Considering the role of $\mathrm{CHCs}$ as contact sex pheromones, our data suggest that host shifts in parasitic wasps might lead to reproductive isolation of host races due to the modification of the cuticular semiochemistry.
\end{abstract}

Keywords: contact sex pheromone, cuticular hydrocarbons (CHCs), host shift, parasitic wasp

(Accepted 29 February 2012; First published online 17 April 2012)

\section{Introduction}

Cuticular hydrocarbons (CHCs) of insects function mainly as a water barrier to avoid desiccation, but also play an important role in intraspecific communication. Because of their low volatility, they act mostly over short distances as contact pheromones (Singer, 1998; Gibbs, 2002; Blomquist \& Bagnères, 2010). CHC profiles are complex mixtures of aliphatic long-chain alkanes and alkenes, as well as methylbranched alkanes. Described functions of $\mathrm{CHCs}$ comprise the mediation of recognition, aggregation, dispersal, alarm and

*Author for correspondence

Fax: +49-9419435583

E-mail: Joachim.ruther@biologie.uni-regensburg.de sexual behaviour in insects (Howard, 1993; Tillmann et al., 1999; Blomquist \& Bagnères, 2010). While social insects also use $\mathrm{CHCs}$ for recognition and interaction with nestmates and as fertility and dominance signals (Singer, 1998; Liebig, 2010), solitary insects mainly use CHCs for the discrimination of conspecifics and enemies, location of mating partners and the elicitation of courtship behaviour (Ruther et al., 2011). Evidence for solitary insects using $\mathrm{CHCs}$ as contact sex pheromones comes from several insect orders, for example the Coleoptera (Buprestidae: Lelito et al., 2009; Silk et al., 2009; Cerambycidae: Ginzel, 2010; Chrysomelidae: Sugeno et al., 2006; Peterson et al., 2007; Geiselhardt et al., 2009), Diptera (Drosophilidae, Glossinidae and Muscidae: Wicker-Thomas, 2007; Ferveur \& Cobb, 2010) and Hymenoptera (Syvertsen et al., 1995; Schiestl et al., 2000; Sullivan, 2002; Mant et al., 2005; Steiner et al., 2005, 2006, 2007). Within the parasitic wasp 
Table 1. Investigated Lariophagus distinguendus strains reared on different beetle hosts.

\begin{tabular}{|c|c|c|c|c|c|c|}
\hline & Host species & Host substrate & Reared on host since & $n \hat{\jmath}$ & $n$ ㅇ & Strain-origin \\
\hline PfoSit & Sitophilus granarius (Curculionidae) & wheat-grains & 2005 & 10 & 14 & Pforzheim \\
\hline StuSteg & Stegobium paniceum (Anobiidae) & koi fish pellets & 2007 & 10 & 15 & Stuttgart \\
\hline BerAca & Acanthoscelides obtectus (Bruchidae) & black-eyed peas & 2008 & 10 & 15 & Berlin \\
\hline BerLas & Lasioderma serricorne (Anobiidae) & wheat-grains & 2004 & 10 & 13 & Berlin \\
\hline
\end{tabular}

$n$, number of samples in chemical analysis.

family Pteromalidae, females of Roptrocerus xylophagorum (Sullivan, 2002), Lariophagus distinguendus (Steiner et al., 2005, 2007), Nasonia vitripennis (Steiner et al., 2006) and Dibrachys cavus (Ruther et al., 2011) produce CHCs which act at short-range as contact sex pheromones eliciting courtship behaviour in males.

The diet of an insect can be an important factor influencing its pheromone communication (Landolt \& Phillips, 1997; Tillmann et al., 1999; Blomquist, 2010). With respect to CHCs, three ways of acquisition are conceivable which are not mutually exclusive: (a) CHCs may be sequestered from the diet after ingestion, (b) absorbed from the environment, or (c) synthesized de novo in oenocytes from dietary precursors (Blomquist \& Jackson, 1973; Etges et al., 2006; Bagnères \& Blomquist, 2010). In the case of parasitic wasps, the first two ways are of particular interest because, due to their parasitic life cycle, these insects have been suggested to have lost the ability to biosynthesize fatty acids (Visser et al., 2010), i.e. the same machinery involved in CHC biosynthesis (Blomquist, 2010). However, the way how parasitic wasps acquire their $\mathrm{CHCs}$ and how the composition is controlled is not well understood. In any case, resources provided by the host should be of crucial importance for the cuticular chemistry.

Because of the influence of diet on the pheromone chemistry of insects, it is reasonable to assume that changes in the diet, e.g. caused by host switches in phytophagous or carnivorous insects, may lead to a breakdown in communication between mating partners and may ultimately contribute to the formation of host races and speciation. In fact, an example for such a host shift-induced breakdown in communication is reported for Drosophila serrata and D. melanogaster. In these species, the development on different substrates was found to induce differences in the $\mathrm{CHC}$ profiles, leading to preferential mating of individuals from the same substrates (Rundle et al., 2005; Sharon et al., 2010). A similar scenario is also thinkable in oligophagous and polyphagous parasitic wasps. Different hosts may provide different pools of precursors for $\mathrm{CHC}$ biosynthesis or different $\mathrm{CHC}$ to be sequestered by the wasps. Consequently, feeding on different hosts might lead to differences in the $\mathrm{CHC}$ profiles of male and female wasps of one population, causing a breakdown in sexual communication and eventually leading to speciation.

As a first step to study this hypothesis, the present paper examines the influence of hosts on the CHC profile in Lariophagus distinguendus, a quasi-gregarious and polyphagous ectoparasitoid of grain infesting beetles (Steidle \& Schöller, 1997). Female cuticular hydrocarbons have been shown to arrest males and elicit wing fanning, a typical element of the male courtship behaviour (Steiner et al., 2005). Interestingly, also pupae of both sexes and newly emerged males elicit courtship behaviour in older males. Unlike females, however, males deactivate the behaviourally active chemicals within $32 \mathrm{~h}$ after emergence (Steiner et al., 2005, 2007; Ruther \& Steiner, 2008). Thus, CHCs evolved to a sexspecific contact pheromone mediating mate recognition in L. distinguendus. We analyzed the CHCs of wasps from six strains reared on four different host species and analyzed the relative composition of the profiles by multivariate statistical methods. Our questions were: Are there differences between the $\mathrm{CHC}$ profiles from strains reared on different hosts? Do these differences occur in both sexes? Which compounds account for the differences in CHC profile? Can differences in the $\mathrm{CHC}$ profiles be caused by a host shift on an alternative host already within one generation? Do the wasps directly sequester significant amounts of host CHCs? The results are discussed with respect to a possible role of $\mathrm{CHCs}$ in prezygotic isolation and sympatric speciation.

\section{Materials and methods}

Insects

Six strains of L. distinguendus were reared on four different beetle species as hosts as described by Steidle \& Schöller (1997) (table 1). The wasp cultures were kept in Petri dishes at $25^{\circ} \mathrm{C}$, L16:D08 photoperiod and 50\% RH. Freshly emerged wasps were isolated and kept under the same conditions for two days. Afterwards, they were deep frozen and stored at $-23^{\circ} \mathrm{C}$ until they were extracted for chemical analysis.

\section{Chemical analysis}

For the analysis of CHCs, three L. distinguendus individuals from the same strain and sex were pooled and extracted for $15 \mathrm{~min}$ in $30 \mu \mathrm{l}$ of hexane containing tetracosane $\left(2.6 \mathrm{ng}^{-1} \mathrm{l}^{-1}\right)$ as an internal standard. The solvent was evaporated under a gentle stream of nitrogen, and the sample was re-dissolved in $10 \mu \mathrm{l}$ of hexane. To investigate the possible sequestration of ingested host-derived CHCs by the parasitoid, we also analyzed the CHC profiles of the four beetle hosts both in the larval and in the adult stage following the protocol described above ( $n=3$ for each host species and stage, respectively). Aliquots ( $1 \mu \mathrm{l}$ in splitless mode) of these extracts were analyzed by coupled gas-chromatography mass spectrometry (GC-MS) on a Shimadzu GCMS-QP2010 Plus quadrupole MS (Shimadzu, Tokyo, Japan) equipped with a $30 \mathrm{~m} \times 0.32 \mathrm{~mm} \mathrm{I}$. D. BPX5 forte capillary column (film thickness $0.25 \mu \mathrm{m}$ ) (SGE Analytical Science Europe, Milton Keynes, UK). Helium was used as carrier gas at a constant column flow of $1.73 \mathrm{ml} \mathrm{min}^{-1}$. The oven program started at $150^{\circ} \mathrm{C}$ and was increased at $3^{\circ} \mathrm{C}$ $\min ^{-1}$ up to $300^{\circ} \mathrm{C}$ (held for $20 \mathrm{~min}$ ). The GC effluent was ionized by electron impact ionization at $70 \mathrm{eV}$; the mass range reached from $\mathrm{m} / \mathrm{z} 35$ to $\mathrm{m} / \mathrm{z} 600$. 
Relative retention indices (LRI) of methyl-branched and unsaturated hydrocarbons were estimated by co-injection of straight-chain hydrocarbons (van Den Dool \& Kratz, 1963). Methyl-branched hydrocarbons were identified by diagnostic ions resulting from the favoured fragmentation at the branching points (Lockey, 1988; Nelson, 1993) and by comparing LRI values with literature data (Carlson et al., 1998; Steiner et al., 2005, 2006, 2007). Positions of the double bonds of unsaturated hydrocarbons were determined by iodine-catalyzed methylthiolation using dimethyl disulphide (Francis \& Velant, 1981; Howard, 1993). MS and LRI data of identified compounds were used to build a custom MS library allowing automatic analysis of GC-MS runs with the help of a two-dimensional search algorithm (MS + LRI) using the GC-MS Solution scientific software (Shimadzu) of the mass spectrometer.

\section{Host shift experiment}

Female wasps from the BerSit strain kept on Sitophilus granarius (F0) were reared for one generation on Stegobium paniceum (F1). The CHC profiles of the wasps from the F1 generation were analyzed as described above. The resulting data were compared to those from female wasps, which were reared at the same conditions but without a host shift (F1 S. granarius).

\section{Statistical analysis}

We integrated the 50 largest peaks (by area) within each run (overlapping compounds were calculated together). All peaks larger than $1 \%$ of the whole peak area were selected for further analysis. The absolute amount of each compound was calculated by relating individual peak areas to the internal standard. Statistical analysis was conducted with PAST version 2.01 scientific software (Hammer et al., 2001). We used the non-metric multidimensional scaling (NMDS, BrayCurtis similarity measure) to visualize the data and the nonparametric MANOVA (NPMANOVA, Bray-Curtis similarity measure of Bonferroni-corrected data) for calculation of the differences between CHC profiles of wasps from the different hosts. Similarity percentage (SIMPER) was used to calculate the individual contribution of each peak to the differences between wasps from different hosts.

\section{Results}

The CHC profiles of the wasps consisted mainly of methylbranched long chain alkanes. For the analysis of female and male profiles, 33 and 30 compounds, respectively, were used (table 2-3). Overall, 83 female and 58 male samples were analyzed. Data of wasps originating from different strains but reared on the same host species (table 1) were pooled for statistical analysis since there were no significant differences in the NPMANOVA analysis between strains reared on the same host-species ( $S$. granarius Berlin vs. Pforzheim: $P=1$ (males), $P=0.9285$ (females); $S$. paniceum Stuttgart vs. Ravensburg: $P=1$ (males); permutation $n=10,000$ ) with the exception of female wasps grown on Stegobium paniceum from the Ravensburg and the Pforzheim strain $(P=0.0045)$.

\section{Differences in CHC profiles of females reared on different host-species}

The NMDS analysis of the CHC profiles of L. distinguendus females reared on different beetle species as hosts (fig. 1A) showed a distinct separation between a cluster consisting of wasps from S. granarius, A. obtectus and L. serricorne and a cluster of wasps from $S$. paniceum. While wasps from S. granarius and A. obtectus overlapped fully, L. serricorne was concentrated at one edge of the cluster. This is also reflected in the NPMANOVA analysis, which gave significant differences between all hosts $(P<0.05$; permutation $n=10,000)$ with the exception of $S$. granarius and $A$. obtectus $(P=0.1566)$. The SIMPER analysis allowed identification of compounds contributing most to the dissimilarity of the $\mathrm{CHC}$ profiles of wasps from different beetle hosts. The compounds with the strongest impact were: 3,7,11,15-tetramethyltritriacontane, 11,21- +11,15-dimethyltritriacontane, 3,7,11-trimethyltritriacontane, and an unknown compound with an LRI of 3089 (table 2).

\section{Differences in CHC profiles of males reared on different host-species}

In L. distinguendus males reared on different beetle species, NMDS indicated also a separation of a cluster consisting of males from S. granarius and A. obtectus and a cluster consisting of males from S. paniceum (fig. 1B). Males from Lasioderma serricorne were located in a third cluster intermediate to the others. While wasps from $S$. granarius and $A$. obtectus overlapped, the other clusters did not. This result was also supported by NPMANOVA analysis, which revealed significant differences between all hosts $(P<0.05$; permutation $n=10,000)$ with the exception of wasps from $S$. granarius and $A$. obtectus $(P=1)$. SIMPER analysis of the male profiles revealed 3,7,11,15-tetramethyltritriacontane, 13,17-dimethylpentatriacontane, 3-methyltritriacontane and 11,21- +11,15dimethyltritriacontane as compounds with major influence on the dissimilarity of the $\mathrm{CHC}$ profiles of wasps from different beetle hosts (table 3).

\section{Host shift experiment}

NMDS-analysis of female wasps (fig. 2) showed a clear separation between clusters formed by CHC profiles of wasps reared on $S$. granarius and $S$. paniceum in the F1 generation. This result was supported by NPMANOVA analysis, which revealed significant differences between both strains $(P<0.05$; permutation $n=10,000)$. The compounds with the highest influence on the dissimilarity of profiles were 3,7,11,15tetramethyltritriacontane, 11,21- +11,15-dimethyltritriacontane, 13,17-dimethylpentatriacontane and the peak belonging to the co-eluting compounds 15- +13- +11-methyltritriacontane (SIMPER analysis; table 4).

\section{Comparison of $\mathrm{CHC}$ profiles from hosts and parasitoid}

To investigate the possible direct sequestration of CHCs from the host into the parasitoid, we compared the $\mathrm{CHC}$ profiles of beetle hosts and the respective parasitoids. These analyses revealed that host $\mathrm{CHCs}$ cannot account for the observed major differences of the $\mathrm{CHC}$ profiles because the $\mathrm{CHC}$ profiles of the wasps are generally composed of compounds with higher molecular masses when compared to 
Table 2. Similarity Percentage (SIMPER) analysis of the L. distinguendus females CHC-profiles (overall average dissimilarity: 29.19).

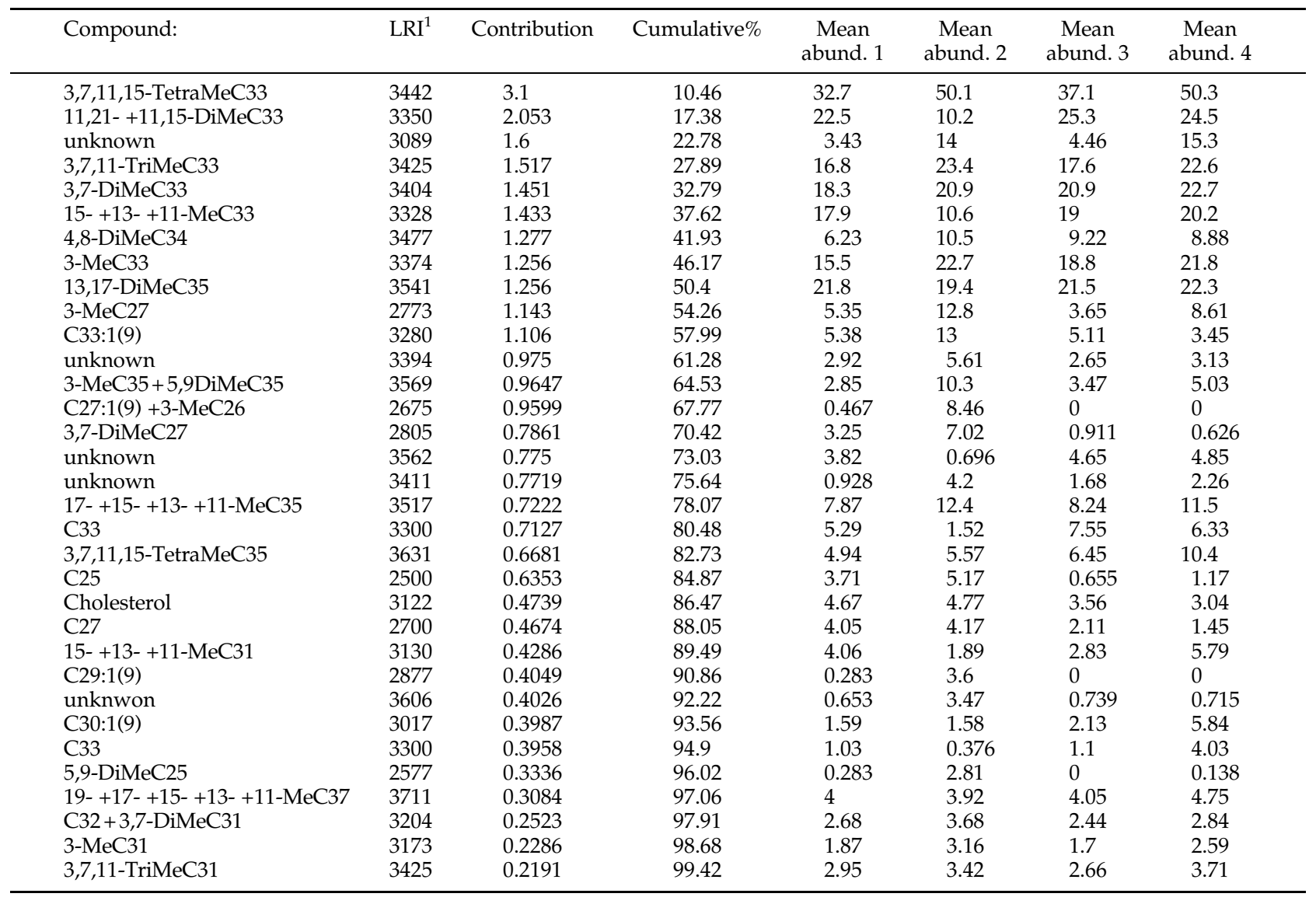

${ }^{1}$ Linear Retention Index according to van Den Dool \& Kratz (1963).

larval and adult stages of the respective beetle hosts (for comparative fingerprint chromatograms see figs S1-4 in the supplementary material). The major compound of the parasitoids are almost absent in the hosts. Conversely, several major compounds of the beetle hosts occurred only in traces in the CHC profiles of the wasps or were completely absent. Apart from A. obtectus, CHCs were hardly present in cuticular extracts from larvae.

\section{Discussion}

The chemical analysis of CHC profiles of female and male L. distinguendus wasps reared on different beetle hosts revealed significant quantitative differences. These were not only observed between strains from different hosts but also between individuals from the same strain which were reared on the two hosts, S. granarius and S. paniceum. In contrast, the profiles of wasp strains reared on the same host species were similar with the exception of female wasps from the Ravensburg and Pforzheim strain reared on S. paniceum. Furthermore, some of the compounds with major influence on the differences between wasp strains reared on S. granarius or S. paniceum were also found to be important in the host-shift experiment. Thus, the CHC profiles of L. distinguendus are indeed host dependent.
Remarkably, the differences in CHC profiles between wasps from $S$. granarius and S. paniceum were present already after one generation and did not require several generations to develop. This indicates that the presence and quantity of compounds in the CHC profiles do not depend on strainrelated features but are presumably caused by host-dependent precursors in the diet of the wasps. Although direct incorporation of host $\mathrm{CHCs}$ can only be demonstrated by labelling experiments (Blomquist \& Jackson, 1973), which, to our knowledge, have never been performed in parasitic wasps, this is unlikely in L. distinguendus. Neither larvae nor adults of the four beetle hosts had significant amounts of the parasitoids' major CHCs on their cuticle, and vice versa many major components of the beetle CHCs were absent from the wasps' cuticle or occurred only in traces. This suggests that the host species influences the wasps' own CHC metabolism rather than serving as a direct source for $\mathrm{CHC}$ sequestration. The published literature on parasitoid/host CHCs does not provide a clear picture of whether parasitoids are able to sequester significant amounts of host CHCs or not. Some species share major components with their hosts (see for instance Howard \& Liang, 1993; Howard \& Infante, 1996); whereas, in other studies, host and parasitoid profiles differed clearly (Howard \& Perez-Lachaud, 2002). Like in the present study, the qualitative composition of the $\mathrm{CHC}$ profile was largely independent from the host in the bethylid wasp 
Table 3. Similarity Percentage (SIMPER) analysis of the L. distinguendus males CHC-profiles (Overall average dissimilarity: 30.92).

\begin{tabular}{|c|c|c|c|c|c|c|c|}
\hline Compound: & $\mathrm{LRI}^{1}$ & Contribution & Cumulative $\%$ & $\begin{array}{l}\text { Mean } \\
\text { abund. } 1\end{array}$ & $\begin{array}{c}\text { Mean } \\
\text { abund. } 2\end{array}$ & $\begin{array}{c}\text { Mean } \\
\text { abund. } 3\end{array}$ & $\begin{array}{c}\text { Mean } \\
\text { abund. } 4\end{array}$ \\
\hline 3,7,11,15-TetraMeC33 & 3442 & 3.927 & 12.7 & 10.5 & 24.2 & 9.22 & 21.2 \\
\hline 13,17-DiMeC35 & 3541 & 3.04 & 22.53 & 15.5 & 25.1 & 12.9 & 16.2 \\
\hline 11,21- +11,15-DiMeC33 & 3351 & 2.474 & 38.73 & 19.9 & 13.1 & 19.2 & 15.8 \\
\hline 3,7,11-TriMeC33 & 3426 & 1.916 & 44.93 & 5.58 & 12.5 & 5.72 & 8.6 \\
\hline unknown & 3567 & 1.854 & 50.92 & 2.25 & 10.4 & 2.41 & 3.73 \\
\hline $\mathrm{C} 33$ & 3300 & 1.294 & 64.97 & 6.36 & 2.19 & 6.21 & 7.27 \\
\hline $17-+15-+13-+11-\mathrm{MeC} 35$ & 3520 & 1.227 & 68.94 & 4.26 & 8.56 & 3.25 & 4.65 \\
\hline unknown & 3396 & 0.8648 & 71.74 & 0.47 & 4.29 & 0.452 & 0.569 \\
\hline unknown & 3126 & 0.8101 & 74.36 & 3.38 & 0.912 & 2.51 & 1.25 \\
\hline $\mathrm{C} 25$ & 2500 & 0.734 & 76.73 & 2.85 & 1.82 & 0.986 & 0.0863 \\
\hline 3,7,11,15-TetraMeC35 & 3632 & 0.6825 & 78.94 & 2.13 & 3.22 & 1.6 & 5 \\
\hline $\mathrm{C} 32$ +3,7-DiMeC31 & 3206 & 0.4105 & 89.51 & 0.74 & 1.91 & 0.354 & 0.0949 \\
\hline $\mathrm{C} 31$ & 3100 & 0.4002 & 90.8 & 2.15 & 0.752 & 2.06 & 1.65 \\
\hline unknown & 3453 & 0.3946 & 92.08 & 0.201 & 0.4 & 0.323 & 2.53 \\
\hline 4,8-DiMeC34 & 3478 & 0.3849 & 93.32 & 2.06 & 2.82 & 2.22 & 2.37 \\
\hline unknown & 3589 & 0.3541 & 94.47 & 0.0913 & 1.72 & 0.153 & 0.331 \\
\hline 3-МeC31 & 3175 & 0.3456 & 95.58 & 1.15 & 2.27 & 0.992 & 0.7 \\
\hline unknown & 3150 & 0.3332 & 96.66 & 1.63 & 0.536 & 1.47 & 0.557 \\
\hline unknown & 2797 & 0.3173 & 97.69 & 1.23 & 0.643 & 1.78 & 0.889 \\
\hline C29 & 2900 & 0.2743 & 98.57 & 1.41 & 0.476 & 1.16 & 0.627 \\
\hline 3247 & 3249 & 0.2208 & 99.29 & 1.33 & 1.07 & 0.822 & 0.703 \\
\hline unknown & 3340 & 0.2204 & 100 & 1.11 & 0.667 & 1.42 & 0.98 \\
\hline
\end{tabular}

${ }^{1}$ Linear Retention Index according to van Den Dool \& Kratz (1963).
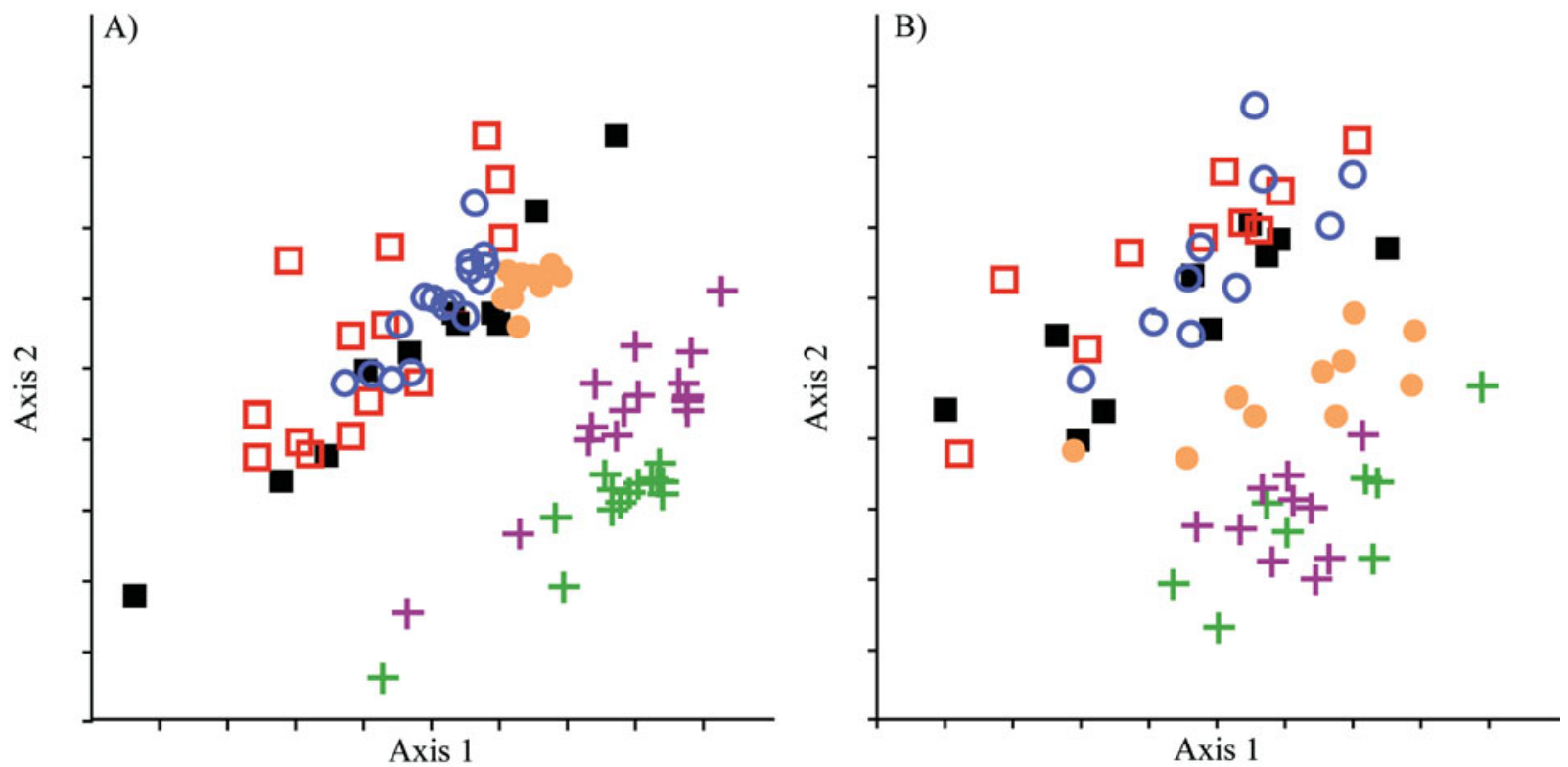

Fig. 1. Non-metric multidimensional scaling (NMDS; Bray-Curtis similarity measure) of the CHC profiles of L. distinguendus females (A) and males (B). Hosts: $\square=$ S. granarius (Pforzheim); घ, S. granarius (Berlin); +, S. paniceum (Ravensburg); +, S. paniceum (Stuttgart); o, A. obtectus (Berlin); •, L. serricorne (Berlin). 
Table 4. Similarity Percentage (SIMPER) analysis of CHC-profiles of L. distinguendus wasps from the host shift experiment (Overall average dissimilarity: 25.76).

\begin{tabular}{|c|c|c|c|c|c|}
\hline Compound: & $\mathrm{LRI}^{1}$ & Contribution & Cumulative $\%$ & Mean abund. 1 & Mean abund. 2 \\
\hline 3,7,11,15-TetraMeC33 & 3443 & 4.507 & 17.49 & 62.1 & 39.9 \\
\hline 11,21- +11,15-DiMeC33 & 3350 & 3.206 & 29.94 & 38.6 & 24 \\
\hline 13,17-DiMeC35 & 3542 & 2.185 & 38.42 & 30.9 & 21.1 \\
\hline 3-MeC33 & 3374 & 1.433 & 51.39 & 24.2 & 17.9 \\
\hline 3,7,11-TriMeC33 & 3425 & 1.412 & 56.87 & 24.5 & 19.1 \\
\hline unknown & 3089 & 1.294 & 61.89 & 10.7 & 4.16 \\
\hline C30:1(9) & 3016 & 0.8262 & 72.42 & 5.74 & 1.37 \\
\hline C33:1(9) & 3280 & 0.8067 & 75.55 & 1.54 & 5.71 \\
\hline 4,8-DiMeC32 & 3285 & 0.6666 & 78.14 & 3.85 & 0.712 \\
\hline 3-MeC27 & 2773 & 0.6603 & 80.7 & 5.99 & 4.48 \\
\hline 17- +15- +13- +11-MeC35 & 3517 & 0.5934 & 83 & 9.96 & 8.16 \\
\hline unknown & 2173 & 0.5399 & 85.1 & 0 & 3.31 \\
\hline unknown & 2801 & 0.2619 & 94.48 & 0.778 & 1.88 \\
\hline unknown & 3394 & 0.2507 & 95.45 & 1.39 & 1.9 \\
\hline $\mathrm{C} 27$ & 2700 & 0.2479 & 96.41 & 1.05 & 2.14 \\
\hline 3,7,11-TriMeC31 & 3227 & 0.2389 & 97.34 & 2.64 & 2.91 \\
\hline $19-+17-+15-+13-+11-\mathrm{MeC} 37$ & 3712 & 0.229 & 98.23 & 3.74 & 3.28 \\
\hline $\mathrm{C} 32+3,7-\mathrm{DiMeC} 31$ & 3204 & 0.1951 & 98.99 & 2.26 & 2.68 \\
\hline 3-MeC31 & 3173 & 0.1365 & 99.52 & 1.84 & 1.93 \\
\hline C29 & 2900 & 0.1247 & 100 & 1.35 & 1.78 \\
\hline
\end{tabular}

${ }^{1}$ Linear Retention Index according to van Den Dool \& Kratz (1963).

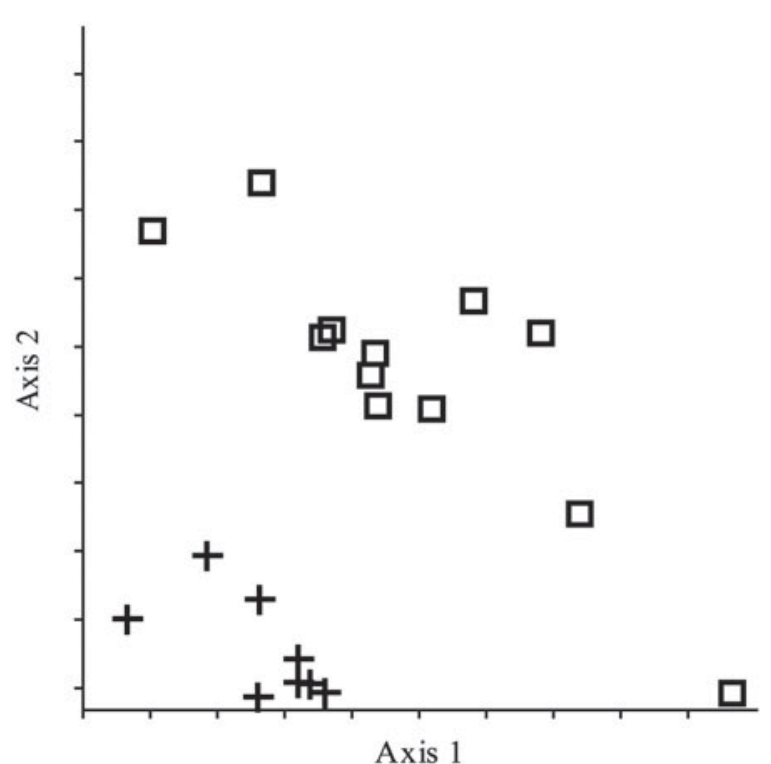

Fig. 2. Host shift experiment: Non-metric multidimensional scaling (NMDS; Bray-Curtis similarity measure) of the CHC profiles of $L$. distinguendus females Hosts: $\square$, S. granarius (BerSit; F1); + , S. paniceum (formerly BerSit; reared for one generation on S. paniceum; F1).

Cephalonomia hyalinipennis and the pteromalid wasp Pteromalus cerealellae, whereas the relative quantities of the components differed (Howard, 2001; Howard \&
Perez-Lachaud, 2002). The biosynthesis of CHCs is closely associated with the fatty acid metabolism. For the synthesis of methyl-branched compounds, considerable amounts of valine, leucine, isoleucine, and methionine are also needed (Blomquist, 2010). These amino acids are among the essential dietary resources for insects, which cannot be biosynthesized de novo by themselves (Behmer, 2006). Hence, differing pools of limiting primary nutrients provided by the different hosts might account for the observed differences in the $\mathrm{CHC}$ profiles.

Interestingly, the CHC profiles of L. distinguendus wasps reared on $A$. obtectus and $S$. granarius overlapped in NMDS analysis, and the $\mathrm{CHC}$ profiles of wasps reared on L. serricorne cluster close to this group and are well separated from the $\mathrm{CHC}$ profiles of wasps reared on S. paniceum. Thus, the $\mathrm{CHC}$ profiles of wasps from these non-related hosts are more similar than the CHC profiles of those reared on L. serricorne and $S$. paniceum, which belong to the same family. It is most likely that S. granarius, A. obtectus and L. serricorne represent similar food substrates and provide qualitative and quantitative similar precursors for the CHCs of L. distinguendus, despite their phylogenetic differences.

In conclusion, our data demonstrate that the composition of CHC profiles in parasitic wasps depend on the host on which the wasps have developed. Because these differences arise already within one generation on a specific host, the composition of the CHC profiles is most likely determined by host-dependent precursors in the diet of the wasps. Since $\mathrm{CHCs}$ are known to play an important role in the recognition of conspecifics and mating partners in these insects (Sullivan, 2002; Steiner et al., 2005, 2006, 2007; Ruther, et al., 2011), it is 
possible that the differences in $\mathrm{CHC}$ profiles caused by different hosts represent a reproductive barrier and may finally contribute to the formation of host-races and eventually to new species. This scenario might be more common in parasitic wasps, which could explain the high diversity in this group of insects. Future studies will have to address the question if the observed effects on the cuticular chemistry actually influence the courtship behaviour of L. distinguendus. Furthermore, it will be interesting to study which differences in host chemistry are responsible for the differences in the CHC profiles of L. distinguendus.

\section{Acknowledgements}

We are grateful to C. Schmid for technical assistance and J. Stökl for his help with statistical analyses. Two anonymous reviewers gave helpful comments on an earlier draft of the manuscript. This research was funded by the Deutsche Forschungsgemeinschaft (DFG, grant RU-717/8-2.). S.K. was supported by a doctoral scholarship of the Universität Bayern e.V.

\section{Text summary of Supplementary Material}

The supplementary material (pdf, $455 \mathrm{~KB}$ ) consists of four figures showing comparative GC-MS chromatograms of cuticular extracts from female Lariophagus distinguendus wasps and the respective hosts (adult and larval stage).

\section{References}

Bagnères, A.G. \& Blomquist, G.J. (2010) Site of synthesis, mechanism of transport and selective deposition of hydrocarbons. pp. 75-99 in Blomquist, G.J. \& Bagnères, A.G. (Eds) Insect Hydrocarbons: Biology, Biochemistry and Chemical Ecology. Cambridge, UK, Cambridge University Press.

Behmer, S.T. (2006) Insect dietary needs: plants as food for insects. pp. 1-4 in Goodman, R.M. (Ed.) Encyclopedia of Plant and Crop Science. New York, USA, Marcel Dekker Publishers.

Blomquist, G.J. (2010) Biosynthesis of cuticular hydrocarbons. pp. 35-52 in Blomquist, G.J. \& Bagnères, A.G. (Eds) Insect Hydrocarbons: Biology, Biochemistry and Chemical Ecology. Cambridge, UK, Cambridge University Press.

Blomquist, G.J. \& Bagnères, A.G. (2010) Introduction: history and overview of insect hydrocarbons. pp. 3-18 in Blomquist, G.J. \& Bagnères, A.G. (Eds) Insect Hydrocarbons: Biology, Biochemistry and Chemical Ecology. Cambridge, UK, Cambridge University Press.

Blomquist, G.J. \& Jackson, L.L. (1973) Incorporation of labelled dietary n-alkanes into cuticular lipids of the grasshopper Melanoplus sanguinipes. Journal of Insect Physiology 19, 1639-1647.

Carlson, D.A., Bernier, U.R. \& Sutton, B.D. (1998) Elution patterns from capillary GC for methyl-branched alkanes. Journal of Chemical Ecology 24, 1845-1865.

Etges, W.J., Veenstra, C.L. \& Jackson, L.L. (2006) Premating isolation is determined by larval rearing substrates in cactophilic Drosophila mojavensis. VII. Effect of larval dietary fatty acids on adult epicuticular hydrocarbons. Journal of Chemical Ecology 32, 2629-2646.

Francis, G.W. \& Velant, K. (1981) Alkylthiolation for the determination of double-bond position in linear alkanes. Journal of Chromatography 219, 379-384.

Ferveur, J.F. \& Cobb, M. (2010) Behavioral and evolutionary roles of cuticular hydrocarbons in Diptera. pp. 325-344 in
Blomquist, G.J. \& Bagnères, A.G. (Eds) Insect Hydrocarbons. Cambridge, UK, Cambridge University Press.

Geiselhardt, S., Otte, T. \& Hilker, M. (2009) The role of cuticular hydrocarbons in male mating behavior of the mustard leaf beetle, Phaedon cochleriae. Journal of Chemical Ecology 35, 1162-1171.

Gibbs, A.G. (2002) Lipid melting and cuticular permeability: new insights into an old problem. Journal of Insect Physiology 48, 391-400.

Ginzel, M.D. (2010) Hydrocarbons as contact pheromones of longhorned beetles. pp. 375-389 in Blomquist, G.J. \& Bagnères, A.G. (Eds) Insect Hydrocarbons. Cambridge, UK, Cambridge University Press.

Hammer, Ø., Harper, D.A.T. \& Ryan, P.D. (2001) Paleontological statistics software package for education and data analysis. Palaeontologia Electronica 4, 9 pp.

Howard, R.W. (1993) Cuticular hydrocarbons and chemical communication. pp. 179-226 in Stanly-Samuelson, D.W. \& Nelson, D.R. (Eds) Insect Lipids. Chemistry, Biochemistry and Biology. Lincoln, NE, USA, University of Nebraska Press.

Howard, R.W. (2001) Cuticular hydrocarbons of adult Pteromalus cerealellae (Hymenoptera: Pteromalidae) and two larval hosts, angoumois grain moth (Lepidoptera: Gelechiidae) and cowpea weevil (Coleoptera: Bruchidae). Annals of the Entomological Society of America 94, 152-158.

Howard, R.W. \& Infante, F. (1996) Cuticular hydrocarbons of the host-specific ectoparasitoid Cephalonomia stephanoderis (Hymenoptera: Bethylidae) and its host the coffee berry borer (Coleoptera: Scolytidae). Annals of the Entomological Society of America 8, 700-709.

Howard, R.W. \& Liang, Y. (1993) Cuticular hydrocarbons of winged and wingless morphs of the ectoparasitoid Choetospila elegans Westwood (Hymenoptera: Pteromalidae) and its host, larval lesser grain borer (Rhyzopertha dominica) (Coleoptera: Bostrichidae). Comparative Biochemistry and Physiology B 106, 407-414.

Howard, R.W. \& Perez-Lachaud, G. (2002) Cuticular hydrocarbons of the ectoparasitic wasp Cephalonomia hyalinipennis (Hymenoptera: Bethylidae) and its alternative host, the stored product pest Caulophilus oryzae (Coleoptera: Curculionidae). Archives of Insect Biochemistry and Physiology 50, 75-84.

Landolt, P.J. \& Phillips, T.W. (1997) Host plant influences on sex pheromone behavior of phytophagous insects. Annual Review of Entomology 42, 371-391.

Lelito, J.P., Boroczky, K., Jones, T.H., Fraser, I., Mastro, V.C., Tumlinson, J.H. \& Baker, T.C. (2009) Behavioral evidence for a contact sex pheromone component of the emerald ash borer, Agrilus planipennis Fairmaire. Journal of Chemical Ecology 35, 104-110.

Liebig, J. (2010) Hydrocarbon profiles indicate fertility and dominance status in ant, bee, and wasp colonies. pp. 245-279 in Blomquist, G.J. \& Bagnères, A.G. (Eds) Insect Hydrocarbons: Biology, Biochemistry and Chemical Ecology. Cambridge, UK, Cambridge University Press.

Lockey, K.H. (1988) Lipids of the insect cuticle: origin composition and function. Comparative Biochemistry and Physiology, Part B: Biochemistry \& Molecular Biology 89B, 595-645.

Mant, J., Brandli, C., Vereecken, N.J., Schulz, S., Francke, W. \& Schiestl, F.P. (2005) Cuticular hydrocarbons as sex pheromone of the bee Colletes cunicularius and the key to its mimicry by the sexually deceptive orchid, Ophrys exaltata. Journal of Chemical Ecology 31, 1765-1787. 
Nelson, D.R. (1993) Methyl-branched lipids in insects. pp. 271-315 in Stanley-Samuelson, D.W. \& Nelson, D.R. (Eds) Insect Lipids: Chemistry, Biochemistry and Biology. Lincoln, NE, USA, University of Nebraska Press.

Peterson, M.A., Dobler, S., Larson, E.L., Juárez, D., Schlarbaum, T., Monsen, K.J. \& Francke, W. (2007) Profiles of the cuticular hydrocarbons mediate male mate choice and sexual isolation between hybridising Chrysochus (Coleoptera: Chrysomelidae). Chemoecology 17, 87-96.

Rundle, H.D., Chenoweth, S.F., Doughty, P. \& Blows, M.W. (2005) Divergent selection and the evolution of signal traits and mating preferences. PLoS Biology 3, 1988-1995.

Ruther, J. \& Steiner, S. (2008) Costs of female odour in males of the parasitic wasp Lariophagus distinguendus (Hymenoptera: Pteromalidae). Naturwissenschaften 95, 547-552.

Ruther, J., Döring, M. \& Steiner, S. (2011) Cuticular hydrocarbons as contact sex pheromone in the parasitoid Dibrachys cavus. Entomologia Experimentalis et Applicata 140, 59-68.

Schiestl, F.P., Ayasse, M., Paulus, H.F., Löfstedt, C., Hansson, B. S., Ibarra, F. \& Francke, W. (2000) Sex pheromone mimicry in the early spider orchid (Ophrys sphegodes): patterns of hydrocarbons as the key mechanism for pollination by sexual deception. Journal of Comparative Physiology A 186, 567-574.

Sharon, G., Segal, D., Ringo, J.M., Hefetz, A., ZilberRosenberg, I. \& Rosenberg, E. (2010) Commensal bacteria play a role in mating preference of Drosophila melanogaster. Proceedings of the National Academy of Sciences of the USA 107, 20051-20056.

Silk, P.J., Ryall, K., Lyons, D.B., Sweeny, J. \& Wu, J. (2009) A contact sex pheromone component of the emerald ash borer Agrilus planipennis Fairmaire (Coleoptera: Buprestidae). Naturwissenschaften 96, 601-608.

Singer, T.L. (1998) Roles of hydrocarbons in the recognition systems of insects. American Zoologist 38, 394-405.

Steidle, L.M. \& Schöller, M. (1997) Olfactory host location and learning in the granary weevil parasitoid Lariophagus distinguendus (Hymenoptera: Pteromalidae). Journal of Insect Behavior 10, 331-342.
Steiner, S., Steidler, J.L.M. \& Ruther, J. (2005) Female sex pheromone in immature insect males-a case of preemergence chemical mimicry? Behavioral Ecology and Sociobiology 58, 111-120.

Steiner, S., Herrmann, N. \& Ruther, J. (2006) Characterization of a female courtship pheromone in the parasitoid Nasonia vitripennis. Journal of Chemical Ecology 32, 2777-2788.

Steiner, S., Mumm, R. \& Ruther, J. (2007) Courtship pheromones in parasitic wasps: comparison of bioactive and incactive cuticular hydrocarbon profiles by discriminant analysis. Journal of Chemical Ecology 32, 825-838.

Sugeno, W., Hori, M. \& Matsuda, K. (2006) Identification of the contact sex pheromone of Gastrophysa atrocyanea (Coleoptera: Chrysomelidae). Applied Entomology and Zoology 41, 296-276.

Sullivan, B.T. (2002) Evidence for a sex pheromone in bark beetle parasitoid Roptrocerus xylophagorum. Journal of Chemical Ecology 28, 1045-1063.

Syvertsen, T.C., Jackson, L.L., Blomquist, G.J. \& Vinson, S.B. (1995) Alkadienes mediating courtship in the parasitoid Cardiochiles nigriceps (Hymenoptera: Braconidae). Journal of Chemical Ecology 21, 1971-1989.

Tillmann, J.A., Seybold, S.J., Jurenka, R.A. \& Blomquist, G.J. (1999) Insect pheromones-an overview of biosynthesis and endocrine regulation. Insect Biochemistry and Molecular Biology 29, 481-514.

van Den Dool, H. \& Kratz, P. (1963) A generalization of the retention index system including linear temperature programmed gas-liquid partition chromatography. Journal of Chromatography 11, 463-471.

Visser, B., Le Lann, C., den Blanken, F.J., Harvey, J.A., van Alphen, J.J.M. \& Ellers, J. (2010) Loss of lipid synthesis as an evolutionary consequence of a parasitic lifestyle. Proceedings of the National Academy of Sciences of the USA 107, 8677-8682.

Wicker-Thomas, C. (2007) Pheromonal communication involved in courtship behavior in Diptera. Journal of Insect Physiology 53, 1089-1100. 